\title{
Sliding Mode Based Dynamic State Estimation for Synchronous Generators in Power Systems
}

\author{
Gianmario Rinaldi ${ }^{1}$, Prathyush P. Menon ${ }^{2}$, Christopher Edwards ${ }^{2}$ and Antonella Ferrara ${ }^{1}$
}

\begin{abstract}
This paper deals with the design of a robust sliding mode observer for dynamic state estimation applied to synchronous generators in power systems. Assuming only the frequency deviation of the generator is measured via Phasor Measurement Units (PMUs), we use a robust sliding mode estimation technique to dynamically reconstruct the rotor angle and the transient voltage. The adopted estimation technique is insensitive to matched bounded uncertainties affecting the dynamics of the synchronous generator. A stability analysis and tuning rules for the observer are also provided. Numerical simulations confirm the validity of the approach.
\end{abstract}

\section{Index Terms}

Observers for nonlinear systems; Variable-structure/sliding-mode control; Power systems.

\section{INTRODUCTION}

$\mathbf{T}$ He area of power systems is facing new challenges due to the rapid development of novel heterogeneous renewable energy based sources and increasing power demands [1]. Supervisory Control And Data Acquisition (SCADA) has been the traditional state estimation technique widely used during the last few decades. This method is based on a steady-state model of the power system and it is characterized by both a slow updating speed (in the order of seconds), and non-synchronous information about the network [2].

More recently, Phasor Measurement Unit (PMU)-based wide-area measurement systems have been proposed to overcome the drawbacks of the SCADA approach [3]. PMUs provide synchronized and faster measurements of real-time voltages, currents and frequency in each area of a power network. By using information gathered by PMUs, it is possible to design more accurate, robust and dynamic state estimators to enhance the monitoring of the power network [4]. In this paper, the word dynamic estimation refers to the ability of these estimators, in a power network context, to reconstruct the generator rotor angle, speed deviation, and transient voltage, in addition to the static states of voltage magnitude and voltage phase angle (see, e.g., [5]).

Many papers have proposed Extended Kalman Filter (EKF)-based dynamic state estimators. For example, in [4], a detailed model of an equivalent generator has been used for the EKF design, and the only unknown input was the excitation voltage of the rotor. In [6] (which can be considered as the continuation of [4]), local and wide-area EKF-based estimation has been proposed. The estimation technique is shown to be robust with respect to two unknown inputs (i.e. the excitation voltage of the rotor and the mechanical torque), and to model parameter variations. Three outputs, frequency, and electrical active and reactive power flows, are required for the proposed estimation strategy. In [5], an EKF has been used with application to a 2-axis-fourth-order state space model of a single generator connected to the grid. More recently, to tackle model uncertainties, derivative-free Kalman Filtering has been adopted in a decentralized fashion [7]. The proposed approach requires voltage and phase measurements to be treated as inputs. In [8], a $\mathrm{H}_{\infty}$ Extended Kalman filter (HEKF) has been proposed with bounded uncertainties such as variations in generator reactances, noise and input disturbances.

Sliding mode-based state estimation can be used to dynamically and robustly reconstruct the unmeasured states of a dynamical system [9]. The main advantages of the sliding mode approach are total insensitivity with respect to bounded uncertainties appearing in the matched channel of the system under observation, and finite-time convergence to zero of the output estimation error [9]. In [10] a first order sliding mode observer has been adopted to estimate the solar array current, measuring only the DC link capacitor voltage, and modeling the device as a Linear Time Invariant (LTI) disturbed system. In [11] and [12] state observers have been designed with application to a nonlinear model of Ćuk converters. In [13] an adaptive gain second order sliding mode observer has been employed to reconstruct the unmeasured capacitor voltage for power converters, measuring only the load current and ensuring robustness with respect to model uncertainties. In [14], by measuring the battery terminal voltage, a sliding mode observer has been employed to track time-varying model parameters of lithium-ion batteries, i.e., the so-called "state-of-health". In [15] a super-twisting sliding mode observer has been employed to estimate the speed of a DC motor, modeled as an LTI system.

\footnotetext{
${ }^{1}$ Gianmario Rinaldi and Antonella Ferrara are with Department of Electrical, Computer and Biomedical Engineering, University of Pavia, Pavia, Italy. gianmario.rinaldioleuniversitadipavia.it, antonella.ferrara@unipv.it.

${ }^{2}$ Prathyush P. Menon and Christopher Edwards are with College of Engineering, Mathematics, and Physical Sciences, University of Exeter, Exeter, United Kingdom. P.M.Prathyush@exeter.ac.uk, C.Edwards@exeter.ac.uk.

This is the final version of the accepted paper included in the IEEE Control Systems Letters, vol.2, no. 4, pp. 785-790, 2018.
} 


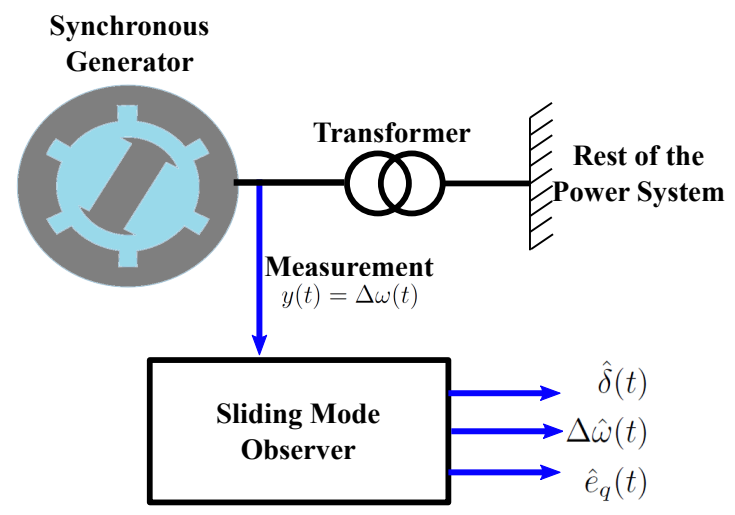

Figure 1. A schematic of a synchronous generator together with the proposed sliding mode observer.

In [16] and [17], a distributed sliding mode observer-based architecture has been adopted to dynamically reconstruct frequency deviation and load voltage phase angles in power systems. In [18], higher order sliding mode observers have been used to reconstruct frequency deviation and power governor variation for a network of thermal power plants. In [19], an observer-based sliding mode control scheme for a single synchronous generator has been proposed to regulate both the speed and voltage of the machine. A nonlinear observer has been employed to estimate excitation and voltage fluxes (the unmeasured state variables) and mechanical torque (the unknown input). Finally, in [20], a multi-variable sliding mode observer has been designed to detect and reconstruct load alterations in a power network, modeled in linear explicit Differential Algebraic Equations (DAEs) form.

Main Contribution: The main contribution of the present paper is the use of a recently proposed sliding mode observer to reconstruct the rotor angle and quadratic-axis transient voltage for synchronous generators in power systems. In [16], [17], and [20], a simplified generator model has been used, neglecting the voltage dynamics. In contrast to the previous approaches, here a nonlinear model accounting for the transient voltage dynamics is adopted. Similar to [6], both excitation voltage and mechanical torque are treated as unknown (bounded) input disturbances. Also similar to [6], [14], and [13] unknown nonlinearities affect the dynamics of the system under observation. However in contrast to [6], in which three outputs (frequency, electrical active and reactive power flows) are required to perform robust dynamic state estimation, in the present paper, only a single measurement output is required, i.e. the frequency deviation, thus reducing the number of required sensors. Specifically, in the proposed approach the synchronous generator is assumed to have nonlinear dynamics around its nominal (stable) working point. We use a sliding mode observer to dynamically reconstruct both the rotor angle and the transient voltage. A proof of stability of the proposed observer is discussed in detail, which makes use of a Lyapunov function technique. Tuning rules for the design constants of the observer are also derived. Numerical simulations are provided to assess the proposed estimation technique.

Structure of the Paper: The rest of the paper is structured as follows. Section II describes the adopted dynamical model of a synchronous generator and introduces the assumptions instrumental for the observer design. Section III presents in detail the design of the sliding mode observer. In Section IV simulation test cases are provided to assess the proposed estimation scheme. Section $\mathrm{V}$ concludes the paper.

Notation: The following (standard) notation is adopted throughout the manuscript. For a state variable $x(t), \hat{x}(t)$ denotes its estimate. For a given matrix $X, X^{T}$ is its transpose. The expression $\operatorname{col}(x, y)$ denotes the column vector $\left[\begin{array}{ll}x & y\end{array}\right]^{T}$. The symbol $I_{a}$ denotes an Identity Matrix of appropriate dimensions. $0_{a b} \in \mathbb{R}^{a \times b}$ denotes a matrix with all zero entries. The expression $\operatorname{sign}(\cdot)$ denotes the signum function. Finally, $\|\cdot\|$ denotes the Euclidean norm. Table I shows a list of adopted symbols. The last column in Table I specifies the numerical values instrumental to Section IV. Figure 1 shows a schematic of a synchronous generator coupled with the proposed sliding mode observer.

\section{Synchronous Generator Model Description}

The so-called third order synchronous generator model is widely used to develop control and monitoring strategies in power systems [3], [21]. This comprises three nonlinear differential equations:

$$
\begin{aligned}
\dot{\delta}(t) & =\omega_{0} \Delta \omega(t) \\
\dot{e}_{q}(t) & =\frac{1}{T_{d 0}}\left(E_{f}(t)-e_{q}(t)-\left(x_{d}-x_{d}^{\prime}\right) \frac{e_{q}(t)-V(t) \cos (\delta(t))}{x_{d}^{\prime}}\right) \\
\Delta \dot{\omega}(t) & =\frac{1}{J}\left(T_{m}(t)-\frac{V(t)}{x_{d}^{\prime}} e_{q}(t) \sin (\delta(t))-\frac{V^{2}(t)}{2}\left(\frac{1}{x_{q}}-\frac{1}{x_{d}^{\prime}}\right) \sin (2 \delta(t))-D \Delta \omega(t)\right) \\
y(t) & =\Delta \omega(t) .
\end{aligned}
$$


Table I

List OF Symbols AND VARIABLES USED IN THE PAPER.

\begin{tabular}{r|c|r}
\hline Symbol & Meaning & Value \\
\hline$\delta(t)$ (rad) & rotor angle & 0.59 \\
$\Delta \omega(t)$ (p.u.) & frequency deviation & 0.00 \\
$V(t)$ (p.u.) & terminal bus voltage & 1.02 \\
$E_{f}(t)$ (p.u.) & excitation voltage & 2.29 \\
$e_{d}(t)$ (p.u.) & d-axis transient voltage & 0.70 \\
$e_{q}(t)$ (p.u.) & q-axis transient voltage & 0.84 \\
$T_{m}(t)$ (p.u.) & mechanical input torque & 0.80 \\
\hline$\omega_{0}$ (rad/s) & frequency nominal value & $120 \pi$ \\
$D$ (p.u.) & damping factor & 0.05 \\
$J$ (p.u.) & inertia constant & 10.00 \\
$T_{d 0}(\mathrm{~s})$ & d-circuit time constant & 0.13 \\
$x_{d}$ (p.u.) & d-axis reactance & 2.06 \\
$x_{q}$ (p.u.) & q-axis reactance & 1.21 \\
$x_{d}^{\prime}$ (p.u.) & d-axis transient reactance & 0.37 \\
\hline
\end{tabular}

Assumption 1 Given the constant (nominal) input signals $\bar{T}_{m}, \bar{E}_{f}, \bar{V}$, there exists an asymptotically stable equilibrium point for the nonlinear system (1). The corresponding constant (nominal) state variables are $\left(\bar{\delta}, \bar{e}_{q}, \Delta \bar{\omega}=0\right)$.

With Assumption 1, the external inputs can be represented as follows $V(t)=\bar{V}+\Delta V(t), E_{f}(t)=\bar{E}+\Delta E_{f}(t), T_{m}(t)=\bar{T}_{m}+$ $\Delta T_{m}(t)$, where $\Delta V(t), \Delta E_{f}(t), \Delta T_{m}(t)$ represent time varying perturbations around the nominal values. Only $\Delta V(t)$ is assumed to be known. For fixed inputs $\bar{T}_{m}, \bar{E}_{f}, \bar{V}$, it is possible to represent (1) as a combination of linear and nonlinear terms as follows:

$$
\begin{aligned}
\dot{X}_{1}(t) & =A_{1} X_{1}(t)+A_{2} X_{2}(t)+G_{1}\left(X_{1}(t), \Delta V(t)\right)+g_{1}\left(E_{f}(t)\right) \\
\dot{X}_{2}(t) & =A_{3} X_{1}(t)+A_{4} X_{2}(t)+G_{2}\left(X_{1}(t), \Delta V(t)\right)+g_{2}(U(t)) \\
y(t) & =X_{2}(t)=C X(t) .
\end{aligned}
$$

where $X(t)=\operatorname{col}\left(X_{1}(t), X_{2}(t)\right)$, and the components $X_{1}(t)=\operatorname{col}\left(\delta(t), e_{q}(t)\right), X_{2}(t)=\Delta \omega(t) ; C=\left[\begin{array}{lll}0 & 0 & 1\end{array}\right] ; G_{1}(\cdot), G_{2}(\cdot)$, $g_{1}(\cdot)$, and $g_{2}(\cdot)$ are properly defined functions, and $U(t)=\operatorname{col}\left(\Delta T_{m}(t), \Delta E_{f}(t), \Delta V(t)\right)$. Only $\Delta \omega(t)$ is measured via the PMUs [3]. The states $\delta(t)$ and $e_{q}(t)$ have to be estimated for the purpose of enhancing the monitoring of the synchronous generator. Matrix $A$, which represents the Jacobian matrix of the nonlinear system (1) about an equilibrium point $\left(\bar{\delta}, \bar{e}_{q}, \Delta \bar{\omega}=0\right)$, can be partitioned as follows

$$
A=\left[\begin{array}{l|l}
A_{1} & A_{2} \\
\hline A_{3} & A_{4}
\end{array}\right]
$$

where $A_{1} \in \mathbb{R}^{2 \times 2}, A_{2} \in \mathbb{R}^{2 \times 1}, A_{3} \in \mathbb{R}^{1 \times 2}$, and $A_{4} \in \mathbb{R}^{1 \times 1}$.

Assumption 2 It is assumed that $G_{1}(\cdot), G_{2}(\cdot)$, and therefore $G(\cdot)=\operatorname{col}\left(G_{1}(\cdot), G_{2}(\cdot)\right)$ are Lipschitz functions with respect to $X_{1}(t)$. Define $\mathcal{L}_{G_{1}}, \mathcal{L}_{G_{2}}, \mathcal{L}_{G}$ as the Lipschitz constants of $G_{1}(\cdot), G_{2}(\cdot)$, and $G(\cdot)$, respectively. It is assumed that $g_{1}(\cdot)$, $g_{2}(\cdot)$, and therefore $g(\cdot)=\operatorname{col}\left(g_{1}(\cdot), g_{2}(\cdot)\right)$ are unknown bounded inputs, with known positive upper bounds on their norms $\Delta_{g_{1}}, \Delta_{g_{2}}$, and $\Delta_{g}$, respectively.

The explicit expression for matrix $A$ associate with (2) can be shown to be

$$
A=\left[\begin{array}{cc|c}
0 & 0 & \omega_{0} \\
\frac{-\bar{V} \sin \bar{\delta}\left(x_{d}-x_{d}^{\prime}\right)}{T_{d 0} x_{d}^{\prime}} & \frac{-x_{d}}{T_{d 0} x_{d}^{\prime}} & 0 \\
\hline-\frac{\bar{V}}{J x_{d}^{\prime}}\left(\bar{V} \frac{x_{d}^{\prime}-x_{q}}{x_{q}} \cos 2 \bar{\delta}+\bar{e}_{q} \cos \bar{\delta}\right) & \frac{-\bar{V} \sin (\bar{\delta})}{J x_{d}^{\prime}} & -\frac{D}{J}
\end{array}\right]
$$

which is Hurwitz, since the equilibrium point $\left(\bar{\delta}, \bar{e}_{q}, \Delta \bar{\omega}=0\right)$ is asymptotically stable. It is possible to determine the Observability Matrix $M_{O}$ associated with the pair $(A, C)$ in (2). For the conditions $\bar{V}=0, \bar{\delta}=0$, and $\bar{e}_{q}=\bar{V}\left(x_{q}-x_{d}^{\prime}\right) / x_{d}$, $\operatorname{det}\left(M_{O}\right)=0$, i.e. $M_{O}$ is not full rank and hence the system is not observable. However, $\bar{V}=0$ and $\bar{\delta}=0$ are unacceptable (voltage magnitude and rotor angle cannot be equal to zero at steady state [3]), whereas $\bar{e}_{q}=\bar{V}\left(x_{q}-x_{d}^{\prime}\right) / x_{d}$ can be acceptable by substituting numerical values in Table I. Recalling the fact that the equilibrium point $\left(\bar{\delta}_{\bar{e}}, \bar{e}_{q}, \Delta \bar{\omega}=0\right)$ is locally asymptotically stable, although $\operatorname{det}\left(M_{O}\right)=0$, the pair $(A, C)$ will be detectable. 


\section{Sliding Mode ObServer Design}

In this section, a robust sliding mode observer is designed in order to estimate the unmeasured state variables. Since $(A, C)$ is detectable, it follows that there exists a matrix $L$ such that $A-L C$ is stable. (Note that $A$ is assumed stable, so the trivial choice $L=0$ can be adopted.) For any $Q>0$ the Lyapunov Equation [22]

$$
(A-L C)^{T} P+P(A-L C)=-Q,
$$

has a unique symmetric positive definite solution $P \succ 0$. Consistent to (3), the matrix $P$ can be partitioned as follows

$$
P=\left[\begin{array}{ll}
P_{1} & P_{2} \\
P_{2}^{T} & P_{3}
\end{array}\right]
$$

where $P_{1} \in \mathbb{R}^{2 \times 2}, P_{2} \in \mathbb{R}^{2 \times 1}$ and $P_{3} \in \mathbb{R}^{1 \times 1}$. Consider a linear change of coordinates $Z(t)=\operatorname{col}\left(Z_{1}(t), Z_{2}(t)\right) \triangleq T X(t)$ for the dynamical system (2), where the matrix $T$ is defined as

$$
T \triangleq\left[\begin{array}{cc}
I_{2} & P_{1}^{-1} P_{2} \\
0_{12} & I_{1}
\end{array}\right]
$$

The change of coordinate is required to obtain a canonical form of the system dynamics for the observer design, in which $\left(A_{1}+P_{1}^{-1} P_{2} A_{3}\right)$ is Hurwitz, exploiting ideas from [23]. The matrix $\tilde{A}=T A T^{-1}$ is given by

$$
\tilde{A}=\left[\begin{array}{c|c}
A_{1}+P_{1}^{-1} P_{2} A_{3} & \begin{array}{c}
A_{2}-A_{1} P_{1}^{-1} P_{2} \\
+P_{1}^{-1} P_{2}\left(A_{4}-A_{3} P_{1}^{-1} P_{2}\right)
\end{array} \\
\hline A_{3} & A_{4}-A_{3} P_{1}^{-1} P_{2}
\end{array}\right]
$$

Consistent with (3), $\tilde{A}$ can also be partitioned into four sub-matrices as follows:

$$
\tilde{A}=\left[\begin{array}{ll}
\tilde{A}_{1} & \tilde{A}_{2} \\
\tilde{A}_{3} & \tilde{A}_{4}
\end{array}\right]
$$

Following [23], by making use of the partition in (9), the system of equations in (2) can be written as

$$
\left.\begin{array}{rl}
\dot{Z}_{1}(t) & =\tilde{A}_{1} Z_{1}(t)+\tilde{A}_{2} Z_{2}(t)+G_{1}(Z(t), \Delta V(t))+g_{1}\left(E_{f}(t)\right)+P_{1}^{-1} P_{2}\left(G_{2}(Z(t), \Delta V(t))+g_{2}(U(t))\right) \\
\dot{Z}_{2}(t) & =\tilde{A}_{3} Z_{1}(t)+\tilde{A}_{4} Z_{2}(t)+G_{2}(Z(t), \Delta V(t))+g_{2}(U(t)) \\
y(t) & =Z_{2}(t) .
\end{array}\right\}
$$

Let the dynamical observer for (10) be:

$$
\left.\begin{array}{rl}
\dot{\hat{Z}}_{1}(t) & =\tilde{A}_{1} \hat{Z}_{1}(t)+\tilde{A}_{2} Z_{2}(t)+G_{1}(\hat{Z}(t), \Delta V(t))+P_{1}^{-1} P_{2}\left(G_{2}(\hat{Z}(t), \Delta V(t))\right) \\
\dot{\hat{Z}}_{2}(t) & =\tilde{A}_{3} \hat{Z}_{1}(t)+\tilde{A}_{4} \hat{Z}_{2}(t)+G_{2}(\hat{Z}(t), \Delta V(t))+v(t) \\
\hat{y}(t) & =\hat{Z}_{2}(t) \\
v(t) & =\left(\left\|\tilde{A}_{4}\right\|\left\|e_{y}(t)\right\|+\rho\right) \operatorname{sign}\left(e_{y}(t)\right)
\end{array}\right\}
$$

where $\hat{Z}_{1}(t)$ is the estimate of $Z_{1}(t), \hat{Z}_{2}(t)$ is the estimate of $Z_{2}(t), \rho$ is a positive design constant, and $v(t)$ is a discontinous injection term depending on the output observation error $e_{y}(t) \triangleq Z_{2}(t)-\hat{Z}_{2}(t)$. The error system dynamics can be obtained subtracting (11) from (10). Specifically, by defining $e_{1}(t) \triangleq Z_{1}(t)-\hat{Z}_{1}(t)$, it follows that

$$
\begin{aligned}
& \dot{e}_{1}(t)=\tilde{A}_{1} e_{1}(t)+\left[I_{2} P_{1}^{-1} P_{2}\right](G(Z(t), \Delta V(t))-G(\hat{Z}(t), \Delta V(t))+g(U(t))) \\
& \dot{e}_{y}(t)=\tilde{A}_{3} e_{1}(t)+G_{2}(Z(t), \Delta V(t))-G_{2}(\hat{Z}(t), \Delta V(t))+g_{2}(U(t))-v(t) .
\end{aligned}
$$

Associate with (12a)-(12b), the following sliding surface:

$$
\mathcal{S}=\left\{\left(e_{1}(t), e_{y}(t)\right) \mid e_{y}(t)=0\right\} .
$$

The following proposition, which represents the main theoretical key-finding of the present paper, will now be proven.

Proposition 1 Given Assumptions 1-2, the error dynamics (12a)-(12b) satisfy the following: $i)\left\|e_{1}(t)\right\|$ remains bounded $\forall t \geq 0$, i.e. $\left\|e_{1}(t)\right\| \leq \beta$, where $\beta$ is a positive constant. ii) The point $e_{1}=0$ is an asymptotically stable equilibrium point for the system (12a) if $g(U(t))=0$ and

$$
\lambda_{\min }\left(Q_{1}\right)>2\left\|\left[\begin{array}{ll}
P_{1} & P_{2}
\end{array}\right]\right\| \mathcal{L}_{G} .
$$


iii) System (12a)-(12b) is driven to the sliding surface (13) in a finite time if the design constant $\rho$ in $v(t)$ is chosen such that

$$
\rho>\left(\left\|\tilde{A}_{3}\right\|+\mathcal{L}_{G_{2}}\right) \beta+\Delta_{g_{2}}+\eta
$$

where $\eta$ is a positive constant.

Proof: i) The generic solution of differential equation (12a) is

$$
e_{1}(t)=\exp \left\{\tilde{A}_{1} t\right\} e_{1}(0)+\int_{0}^{t}\left(\operatorname { e x p } \{ \tilde { A } _ { 1 } ( t - \tau ) \} \left(\left[I_{2} P_{1}^{-1} P_{2}\right](G(Z(\tau), \Delta V(\tau))-G(\hat{Z}(\tau), \Delta V(\tau))+g(U(\tau))) d \tau,\right.\right.
$$

in which $\tilde{A}_{1}$ is Hurwitz. Given the linear change of coordinates (7), it is obvious that $G(\cdot)$ is Lipschitz function with respect to $Z(t)$, and there exist positive constants $a_{0}$ and $c_{0}$ such that [23]

$$
\left\|e_{1}(t)\right\| \leq c_{0} \exp \left\{-a_{0} t\right\}\left\|e_{1}(0)\right\|+\int_{0}^{t} c_{0} \exp \left\{-a_{0}(t-\tau)\right\}\left\|\left[I_{2} \quad P_{1}^{-1} P_{2}\right]\right\|\left(\mathcal{L}_{G}\left\|e_{1}(\tau)\right\|+\Delta_{g}\right) d \tau .
$$

Multiplying both sides of (17) by $\exp \left\{a_{0} t\right\}$,

$$
\left.\exp \left\{a_{0} t\right\}\left\|e_{1}(t)\right\| \leq c_{0}\left(\left\|e_{1}(0)\right\|+\mathcal{L}_{G}\left\|\left[I_{2} \quad P_{1}^{-1} P_{2}\right]\right\| \int_{0}^{t} \exp \left\{a_{0} \tau\right)\right\}\left\|e_{1}(\tau)\right\| d \tau\right)+\frac{c_{0}}{a_{0}} \Delta_{g}\left\|\left[I_{2} \quad P_{1}^{-1} P_{2}\right]\right\|\left(\exp \left\{a_{0} t\right\}-1\right) .
$$

By making use of the Grownwall-Bellman Inequality [23], if

$$
y(t) \leq \lambda(t)+\int_{0}^{t} \mu(\tau) y(\tau) d \tau
$$

then

$$
y(t) \leq \lambda(t)+\int_{0}^{t} \lambda(\tau) \mu(\tau) \exp \left\{\int_{\tau}^{t} \mu(s) d s\right\} d \tau .
$$

Exploiting the structure of equation (18), if

$$
\begin{aligned}
y(t) & \triangleq \exp \left\{a_{0} t\right\}\left\|e_{1}(t)\right\| \\
\mu & \triangleq c_{0} \mathcal{L}_{G}\left\|\left[\begin{array}{ll}
I_{2} & P_{1}^{-1} P_{2}
\end{array}\right]\right\| \\
\lambda(t) & \triangleq c_{0}\left\|e_{1}(0)\right\|+\frac{c_{0}}{a_{0}} \Delta_{g}\left\|\left[I_{2} \quad P_{1}^{-1} P_{2}\right]\right\|\left(\exp \left\{a_{0} t\right\}-1\right),
\end{aligned}
$$

then (18) has the form of (19a). Then from (19b), and after straightforward algebraic manipulations, one obtains

$$
\begin{aligned}
\left\|e_{1}(t)\right\| \leq & c_{0}\left\|e_{1}(0)\right\| \exp \left\{\left(\mu-a_{0}\right) t\right\}+\frac{c_{0}}{a_{0}} \Delta_{g}\left\|\left[\begin{array}{ll}
I_{2} & P_{1}^{-1} P_{2}
\end{array}\right]\right\|\left(1-\exp \left\{-a_{0} t\right\}\right) \\
& +\frac{c_{0}^{2}}{a_{0}} \Delta_{g} \mu\left(\frac{\exp \left\{-a_{0} t\right\}}{\mu}+\frac{1}{\left(a_{0}-\mu\right)}-\exp \left\{\left(\mu-a_{0}\right) t\right\} \frac{1}{\mu\left(a_{0}-\mu\right)}\right) .
\end{aligned}
$$

From (20) it is apparent that

$$
\left\|e_{1}(t)\right\| \leq \beta, \forall t \geq 0, \text { if } \mu=c_{0} \mathcal{L}_{G}\left\|\left[\begin{array}{ll}
I_{2} & P_{1}^{-1} P_{2}
\end{array}\right]\right\|<a_{0},
$$

which proves i).

Remark 1 Note that [23] considers the special case when $\Delta_{g}=0$. A theoretical novelty of the proposed approach, compared to [23], is that the term $\left\|e_{1}(t)\right\|$ remains bounded even in the presence of time varying bounded unknown inputs.

ii) To prove the asymptotic stability of the point $e_{1}=0$, as in [23], the Lyapunov function $V_{e_{1}}=e_{1}(t)^{T} P_{1} e_{1}(t)$ is chosen. The time derivative of $V_{e_{1}}$ yields

$$
\dot{V}_{e_{1}}=e_{1}(t)^{T}\left(P_{1} \tilde{A}_{1}+\tilde{A}_{1}^{T} P_{1}\right) e_{1}(t)+2 e_{1}(t)^{T}\left[P_{1} P_{2}\right](G(Z(t), \Delta V(t))-G(\hat{Z}(t), \Delta V(t))) .
$$

In [23] it has been shown that $P_{1} \tilde{A}_{1}+\tilde{A}_{1}^{T} P_{1}=-Q_{1}$. Then, according to Assumption 2, the following inequalities can be obtained

$$
\dot{V}_{e_{1}} \leq-\left(\lambda_{\min }\left(Q_{1}\right)+2\left\|\left[\begin{array}{ll}
P_{1} & P_{2}
\end{array}\right]\right\| \mathcal{L}_{G}\right)\left\|e_{1}(t)\right\|^{2} .
$$

It follows $\dot{V}_{e_{1}}$ is strictly negative if

$$
\lambda_{\min }\left(Q_{1}\right)>2\left\|\left[\begin{array}{ll}
P_{1} & P_{2}
\end{array}\right]\right\| \mathcal{L}_{G},
$$

which proves ii). 
iii) From (12b), it follows

$$
\begin{aligned}
e_{y}(t)^{T} \dot{e}_{y}(t) & =e_{y}(t)^{T}\left(\tilde{A}_{3} e_{1}(t)+\tilde{A}_{4} e_{y}(t)+G_{2}(Z(t), \Delta V(t))\right. \\
& \left.-G_{2}(Z(t), \Delta V(t))+g_{2}(U(t))-v(t)\right) e_{y}(t)
\end{aligned}
$$

By making use again of Assumption 2, the following inequalities can be obtained

$$
\begin{aligned}
e_{y}(t)^{T} \dot{e}_{y}(t) & \leq\left(\left(\left\|\tilde{A}_{3}\right\|+\mathcal{L}_{G_{2}}\right)\left\|e_{1}(t)\right\|+\Delta_{g_{2}}-\rho\right)\left\|e_{y}(t)\right\| \\
& \leq\left(\left(\left\|\tilde{A}_{3}\right\|+\mathcal{L}_{G_{2}}\right) \beta+\Delta_{g_{2}}-\rho\right)\left\|e_{y}(t)\right\| .
\end{aligned}
$$

The reachability condition [9] is fulfilled if

$$
\rho>\left(\left\|\tilde{A}_{3}\right\|+\mathcal{L}_{G_{2}}\right) \beta+\Delta_{g_{2}}+\eta,
$$

which guarantees reaching of the sliding surface and proves iii).

Note that condition $e_{y}(t)=0$ is reached in a finite time $t_{r}$ which is upper-bounded as follows [9]:

$$
t_{r}<\frac{2\left\|e_{y}(0)\right\|}{\eta} .
$$

In order to evaluate the performance of the sliding mode estimation scheme, it is possible to define the following performance metric $\mathcal{E}(t)$

$$
\mathcal{E}(t) \triangleq \int_{t_{r}}^{t}\left\|e_{y}(\tau)\right\| d \tau
$$

where $t_{r}$ is the time instant at which the sliding motion $e_{y}(t)=0$ occurs. According to the theoretical developments in this section, it is expected that $\mathcal{E}(t)$ is almost zero. This will be shown in simulation environment in Section IV.

Remark 2 Suppose that a differentiable band-limited measurement noise $\psi(t)$ affects the output of the system (10) as $y(t)=$ $Z_{2}(t)+\psi(t)$. In this situation the output estimation error becomes $e_{y}(t) \triangleq Z_{2}(t)+\psi(t)-\hat{Z}_{2}(t)$. Thus, measurement noise affects the proposed observer in (11) (specifically, signals $y(t)$ and $v(t)$ are affected). By exploiting (12a)-(12b), it is apparent that the effect of the noise can be incorporated as part of the unknown bounded input $g(U(t))$, by reasonably increasing the value for $\Delta_{g}$ in Assumption 2. Therefore, part $\left.i\right)$ of Proposition 1 still holds, thus preserving the bounded features of $\left\|e_{1}(t)\right\|$. As for part iii), the sliding motion $e_{y}(t)=0$ still takes place in a finite time. However, as stated above, $e_{y}(t)$ is affected by the measurement noise. By defining the noise-free output error as follows $\check{e}_{y}(t) \triangleq Z_{2}(t)-\hat{Z}_{2}(t)$, one has in a finite time $\hat{Z}_{2}(t)=Z_{2}(t)+\psi(t)$, analogously to [24].

\section{Numerical Test Cases}

In this section numerical test cases are provided in order to assess the proposed sliding mode observer. A single synchronous generator connected to the grid is considered. The model parameters are shown in Table I (base power 1000 (MVA)) together with the nominal equilibrium values for both the state variables and input signals. The simulation time horizon was set equal to 10 (s) and the synchronous generator was modeled in the Matlab-Simulink R2017b environment by using the Odel-Euler solver with an integration step size $\tau=1 \times 10^{-4}$ seconds. The (Hurwitz) Jacobian Matrix $A$ in (4) evaluated for the equilibrium data in Table I is

$$
A=\left[\begin{array}{ccc}
0 & 0 & 377.000 \\
-20.100 & -42.830 & 0 \\
-0.180 & -0.160 & -0.005
\end{array}\right]
$$

For the Lyapunov Equation (5), matrix $Q=I_{3}$, and the gain matrix $L$ is $L=\left[\begin{array}{lll}376.0877 & -173.7180 & -0.0124\end{array}\right]^{T}$. The unique symmetric positive definite solution $P$ is given by

$$
P=\left[\begin{array}{ccc}
10.8153 & -7.2107 & -0.5481 \\
-7.2107 & 19.2845 & 3.9179 \\
-0.5481 & 3.9179 & 0.9121
\end{array}\right]
$$

The design constant $\rho=0.5$. Computing the value of matrix $T$ as in (7), matrix $\tilde{A}$ for the implementation of the observer (11) is obtained as follows:

$$
\tilde{A}=T A T^{-1}=\left[\begin{array}{ccc}
-0.020 & -0.017 & 377.000 \\
-20.125 & -42.866 & -0.001 \\
-0.180 & -0.160 & -0.005
\end{array}\right]
$$



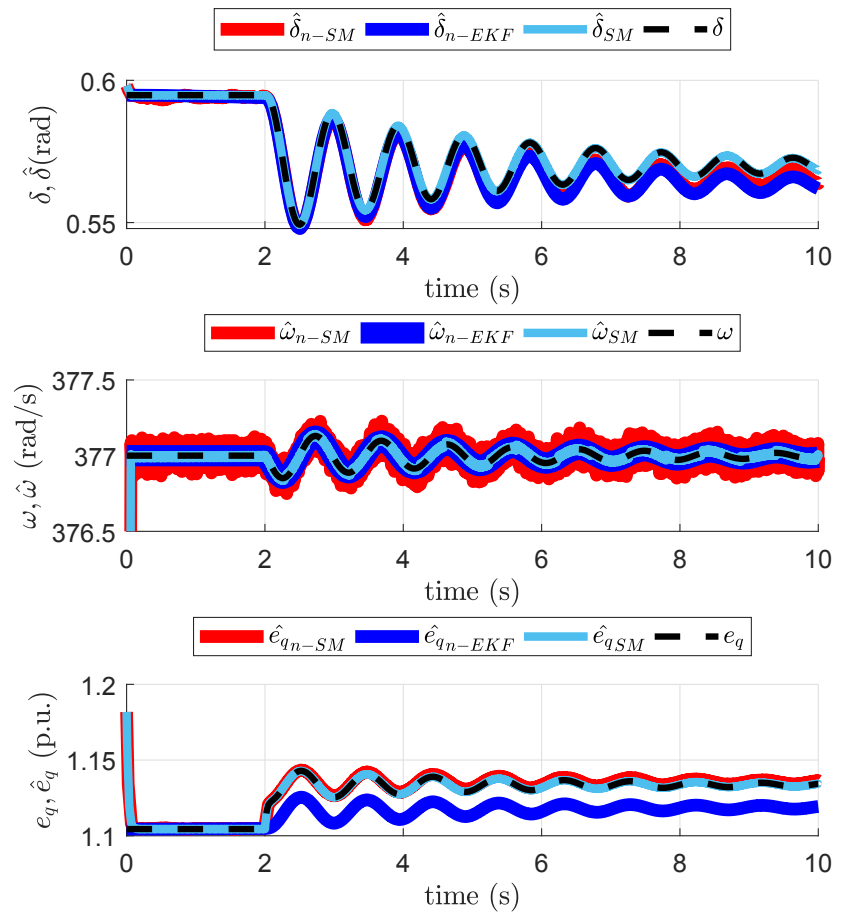

Figure 2. Scenario 1, from the top: time evolution of the rotor angle and its estimates; time evolution of the frequency deviation and its estimates; time evolution of the q-axis transient voltage and its estimates.
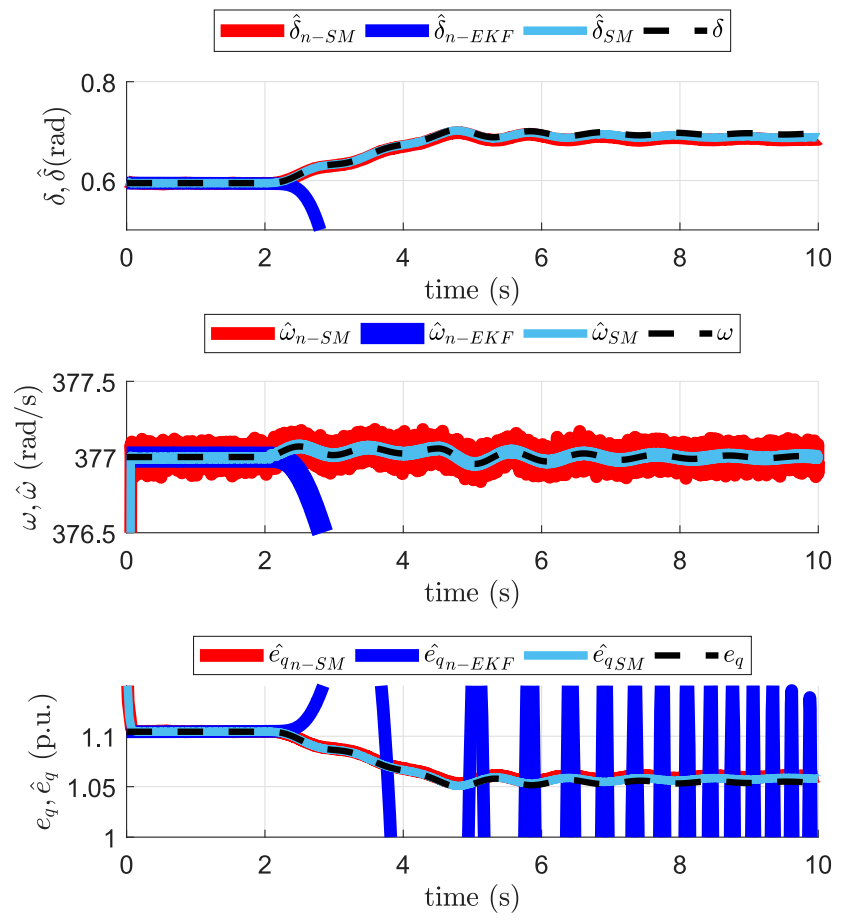

Figure 3. Scenario 2, from the top: time evolution of the rotor angle and its estimates; time evolution of the frequency deviation and its estimates; time evolution of the q-axis transient voltage and its estimates 
Table II

Performance Metric $\mathcal{E}(T)$ in (29) as a Functions of the Variance $\sigma^{2}$ of the Noise $\psi(t), T=10$ Seconds, $(\mu p . u$. $)=(1 e-6 p . u$. $)$, Scenario 1

\begin{tabular}{c|r|r|r|r|r|r|r}
$\sigma^{2}$ & 0.00 & 0.02 & 0.04 & 0.06 & 0.08 & 0.10 & $(\text { р.и. })^{2}$ \\
\hline $\mathcal{E}(T)$ & 225 & 509 & 883 & 1259 & 1637 & 2026 & $($ нр.u. $)$
\end{tabular}

Two scenarios are considered:

1) Scenario 1, during which a step variation of 0.1 (p.u.) takes place in the armature voltage. This means that $E_{f}(t)=$ $2.29+0.1 \cdot \operatorname{step}(t-2)$. Note that this scenario has also been considered in [4].

2) Scenario 2, during which the mechanical input torque is time-varying. Specifically:

$$
T_{m}(t)= \begin{cases}0.8 & 0<t \leq 2 \\ 0.8+0.05 \cdot(t-2) & 2<t \leq 4 \\ 0.9 & 4<t \leq 10\end{cases}
$$

Note that both the input disturbances in the two scenario are unknown by the observer. For the two scenarios, three estimation schemes are considered. Specifically, if $x$ is a state variables, $\hat{x}_{S M}$ denotes its estimate via the sliding mode observer in a measurement noise-free condition, $\hat{x}_{n-S M}$ is used for the estimate obtained via a sliding mode observer with measurement noise, while $\hat{x}_{n-E F K}$ is used for the estimate from an Extended Kalman Filter (EKF) with measurement noise. The variance of the measurement noise is chosen as in [4] to be equal to $\sigma^{2}=0.1$. From the analysis of Figures 2-3, one can conclude that the proposed sliding mode estimation scheme can profitably estimate the unmeasured state variables in the noise free case, but also in presence of measurement disturbances. It is apparent that the EKF attenuates better than sliding mode observer the effect of the measurement noise as shown in Figure 3. However, the EKF performance can degradate in the presence of unknown inputs affecting the system. The evolution of the performance metric $\mathcal{E}(T)$ in (29) as function of the variance of the measurement noise $\sigma^{2}$ is almost linear, as shown in in Table II.

\section{CONCLUSION}

In this paper, a robust sliding mode observer has been designed for dynamic state estimation applied to synchronous generators in power systems. In contrast to the widely used EKF, the sliding mode-based estimation technique applied in this framework is revealed to be robust and totally insensitive to unknown bounded matched uncertainties and requires only one measurement output. The stability properties and the tuning rules of the observer are also provided. Numerical simulations have been used to assess our approach.

\section{REFERENCES}

[1] P. Komarnicki, P. Lombardi, and Z. Styczynski, "Future power systems," in Electric Energy Storage Systems. Springer, 2017, pp. 1-35.

[2] A. Abur and A. G. Exposito, Power System State Estimation: Theory and Implementation. CRC press, 2004.

[3] P. W. Sauer, M. Pai, and J. H. Chow, Power System Dynamics and Stability: With Synchrophasor Measurement and Power System Toolbox. John Wiley \& Sons, 2017

[4] E. Ghahremani and I. Kamwa, "Dynamic state estimation in power system by applying the extended Kalman filter with unknown inputs to phasor measurements," IEEE Transactions on Power Systems, vol. 26, no. 4, pp. 2556-2566, 2011.

[5] H. Tebianian and B. Jeyasurya, "Dynamic state estimation in power systems: Modeling, and challenges," Electric Power Systems Research, vol. 121, pp. $109-114,2015$.

[6] E. Ghahremani and I. Kamwa, "Local and wide-area PMU-based decentralized dynamic state estimation in multi-machine power systems," IEEE Transactions on Power Systems, vol. 31, no. 1, pp. 547-562, 2016.

[7] G. Anagnostou and B. C. Pal, "Derivative-free Kalman filtering based approaches to dynamic state estimation for power systems with unknown inputs," IEEE Transactions on Power Systems, vol. 33, no. 1, pp. 116-130, 2018.

[8] J. Zhao, "Dynamic state estimation with model uncertainties using $H_{\infty}$ Extended Kalman Filter," IEEE Transactions on Power Systems, vol. 33, no. 1 , pp. 1099-1100, 2018.

[9] C. Edwards and S. Spurgeon, Sliding Mode Control: Theory and Applications. CRC Press, 1998.

[10] I.-S. Kim, M.-B. Kim, and M.-J. Youn, "New maximum power point tracker using sliding-mode observer for estimation of solar array current in the grid-connected photovoltaic system," IEEE Transactions on Industrial Electronics, vol. 53, no. 4, pp. 1027-1035, 2006.

[11] A. Pyrkin, R. Ortega, A. Bobtsov, S. Aranovskiy, and D. Gerasimov, "Ćuk converter full state adaptive observer design," in Proc IEEE Conference on Control Applications (CCA), Sydney, Australia, September 2015, pp. 1254-1259.

[12] R. Ortega, A. Bobtsov, A. Pyrkin, and S. Aranovskiy, "A parameter estimation approach to state observation of nonlinear systems," Systems \& Control Letters, vol. 85, pp. 84-94, 2015.

[13] J. Liu, S. Laghrouche, M. Harmouche, and M. Wack, "Adaptive-gain second-order sliding mode observer design for switching power converters," Control Engineering Practice, vol. 30, pp. 124-131, 2014.

[14] I.-S. Kim, "A technique for estimating the state of health of lithium batteries through a dual-sliding-mode observer," IEEE Transactions on Power Electronics, vol. 25, no. 4, pp. 1013-1022, 2010.

[15] J. Rivera, I. Dueñas, S. Ortega, and J. Del Valle, "Field-programmable analogue arrays for the sensorless control of DC motors," International Journal of Electronics, vol. 105, no. 2, pp. 191-210, 2018.

[16] G. Rinaldi, P. P. Menon, C. Edwards, and A. Ferrara, "Distributed observers for state estimation in power grids," in Proc. American Control Conference, Seattle, WA, USA, May 2017, pp. 5824-5829. 
[17] G. Rinaldi and A. Ferrara, "Higher order sliding mode observers and nonlinear algebraic estimators for state tracking in power networks," in Proc. 56th IEEE Conference on Decision and Control, Melbourne, Australia, December 2017, pp. 6033-6038.

[18] G. Rinaldi, M. Cucuzzella, and A. Ferrara, "Third order sliding mode observer-based approach for distributed optimal load frequency control," IEEE Control Systems Letters, vol. 1, no. 2, pp. 215-220, 2017.

[19] J. Cabrera-Vázquez, A. G. Loukianov, J. M. Cañedo, and V. I. Utkin, "Robust controller for synchronous generator with local load via VSC," International Journal of Electrical Power \& Energy Systems, vol. 29, no. 4, pp. 348-359, 2007.

[20] C. Mellucci, P. P. Menon, C. Edwards, and A. Ferrara, "Second-order sliding mode observers for fault reconstruction in power networks," IET Control Theory \& Applications, vol. 11, no. 16, pp. 2772-2782, 2017.

[21] J. Machowski, J. Bialek, and J. R. Bumby, Power System Dynamics and Stability. John Wiley \& Sons, 1997.

[22] H. K. Khalil, Noninear systems. Prentice-Hall, New Jersey, 1996.

[23] X.-G. Yan and C. Edwards, "Robust sliding mode observer-based actuator fault detection and isolation for a class of nonlinear systems," International Journal of Systems Science, vol. 39, no. 4, pp. 349-359, 2008.

[24] A. Poznyak, "Stochastic output noise effects in sliding mode state estimation," International Journal of Control, vol. 76, no. 9-10, pp. 986-999, 2003. 